\begin{tabular}{|c|c|c|}
\hline Beitr. Ent. & Keltern & ISSN 0005-805X \\
\hline $\mathbf{5 7}(2007) 1$ & S. $211-239$ & 30.06 .2007 \\
\hline
\end{tabular}

\title{
Nuovi dati faunistici e tassonomici su specie di Aleocharinae del Sudamerica delle tribù Gyrophaenini, Placusini, Diestotini, Homalotini, Bolitocharini, Paglini e Falagriini ${ }^{1}$
}

\section{(Coleoptera, Staphylinidae)}

Con 88 figure

\section{Roberto Pace}

\begin{abstract}
Summary
Results of taxonomic and faunistic studies on the 67 specimens in the Zoological Museum of the Humboldt University, Berlin, the Deutsches Entomologisches Institut, Müncheberg and the Herbert Franz Collection in the Naturhistorisches Museum, Vienna, are presented. Seven tribes (Gyrophaenini, Placusini, Diestotini, Homalotini, Bolitocharini, Paglini and Falagriini), 14 genera (Gyrophaena, Placusa, Euvira, Diestota, Eudera, Plesiomalota, Tropocoenonica, Prosoponotha, Dispachyglossa, Thecturota, Habramicrada, Pagla, Falagria and Meronera) and 36 species are recognized from South America. Three genera (Tropocoenonica of the Homalotini, Dispachyglossa and Habramicrada of the Bolitocharini), 1 subgenus (Parallelopagla of Pagla) and 28 species are described as new to science. All new descriptions are accompanied by drawings of whole insects and morphological details.
\end{abstract}

Key words

Coleoptera, Staphylinidae, Aleocharinae, taxonomy, South America, Key to Subgenera

\section{Zusammenfassung}

Ergebnisse taxonomischer und faunistischer Untersuchungen an 67 Exemplaren aus dem Zoologischen Museum der Humboldt Universität zu Berlin, aus dem Deutschen Entomologischen Institut, Müncheberg und der Sammlung Herbert Franz im Naturhistorischen Museum, Wien werden dargestellt. 36 südamerikanische Arten in 14 Gattungen (Gyrophaena, Placusa, Euvira, Diestota, Eudera, Plesiomalota, Tropocoenonica, Prosoponotha, Dispachyglossa, Thecturota, Habramicrada, Pagla, Falagria und Meronera), und 7 Tribus (Gyrophaenini, Placusini, Diestotini, Homalotini, Bolitocharini, Paglini und Falagriini) wurden determiniert. Darunter wurden 28 neue Arten beschrieben und 3 neue Gattungen (Tropocoenonica von den Homalotini, Dispachyglossa und Habramicrada der Bolitocharini) sowie 1 neue Untergattung (Parallelopagla von Pagla) aufgestellt. Zu sämtlichen Neubeschreibungen werden Habitus- und Detailzeichnungen beigefügt.

\section{Riassunto}

Sono forniti dati tassonomici e faunistici di 67 esemplari del Museo Zoologico dell'Università Humboldt di Berlino, del Deutsches Entomologisches Institut di Müncheberg e della collezione di Herbert Franz al Naturhistorisches Museum di Vienna. Sono riconosciuti 7 tribù (Gyrophaenini, Placusini, Diestotini,

${ }^{1} 219^{\circ}$ Contributo alla conoscenza delle Aleocharinae. 
Homalotini, Bolitocharini, Paglini e Falagriini), 14 generi (Gyrophaena, Placusa, Euvira, Diestota, Eudera, Plesiomalota, Tropocoenonica, Prosoponotha, Dispachyglossa, Thecturota, Habramicrada, Pagla, Falagria e Meronera) e 36 specie. Sono descritti come nuovi per la scienza tre generi (Tropocoenonica degli Homalotini, Dispachyglossa e Habramicrada dei Bolitocharini), un sottogenere (Parallelopagla di Pagla) e 28 specie. Tutte le nuove specie, i nuovi generi e il nuovo sottogenere sono illustrati. Le nuove specie e i nuovi generi sono confrontati con taxa affini.

\section{New species}

Gyrophaena nemoralis n. sp., Placusa iguazuensis n. sp., Placusa macrocercorum n. sp., Placusa brasiliensis n. sp., Euvira franzi n. sp., Euvira aspera n. sp., Diestota (Apheloglossa) melanuroides n. sp., Diestota (Apheloglossa) elegans n. sp., Diestota (Apheloglossa) dasyporum n. sp., Diestota (Apheloglossa) mixta n. sp., Plesiomalota (Microchara) boliviana n. sp., Plesiomalota (Microusa) belemensis n. sp., Plesiomalota (Microusa) minima n. sp., Plesiomalota (Microusa) surinamensis n. sp., Plesiomalota (Plesiomalota) tarapotensis n. sp., Plesiomalota (Plesiomalota) iguazuensis n. sp., Plesiomalota (Plesiomalota) bradyporum n. sp., Tropocoenonica n. gen., Tropocoenonica catharinensis n. sp., Prosoponotha brasiliensis n. sp., Dispachyglossa n. gen., Dispachyglossa elegans n. sp., Thecturota maculata n. sp., Habramicrada n. gen., Habramicrada boliviensis n. sp., Habramicrada anularis n. sp., Habramicrada peruviana n. sp., Habramicrada incaica n. sp., Pagla (Parallelopagla) n. subgen., Pagla (Parallelopagla) levis n. sp., Falagria (Leptagria) boliviana n. sp.

\section{Introduzione}

È ancora da scrivere una storia naturale della sottofamiglia Aleocharinae del Sudamerica. Nell'ovvia impossibilità di un'ampia trattazione con l'illustrazione di tutte le serie tipiche delle specie sudamericane note, in gran parte da me già esaminate, qui mi limito ad elencare il materiale indeterminato conservato nelle collezioni museali e quello di recenti raccolte. È ovvio che gli esemplari delle collezioni sono una minima parte della massa realmente esistente in natura. Quando moltissime specie e generi saranno descritti e tassonomicamente bene collocati si potrà affrontare la classificazione e lo studio dell'evoluzione a livello mondiale più alti delle specie. Questi studi insieme a quelli di biogeografia, hanno bisogno di molti nuovi lavori (LAWRENCe \& Newton 1982).

Esemplari di Aleocharinae delle regioni australi, fino a tempi recenti, sono stati solamente inadeguatamente ed occasionalmente raccolti, forse a causa della piccola taglia (approssimativamente 0,8-6 mm) di molte specie. Metodi specializzati sono necessari per il loro rinvenimento. I reperti fossili dimostrano che quello degli Stafilinidi è un antico gruppo, già presente e bene diversificato dalla fine del Periodo Giurassico, prima della significativa separazione per deriva delle masse terrestri meridionali, (Tiкhomirova 1968; Arnoldi e altri, 1977). Ciò può spiegare l'odierno gran numero di generi e specie di Aleocharinae nel Globo, circa 1740 generi e 12.182 specie finora noti (Herman, 2001), destinato ad un impressionante accrescimento quando si progetteranno lavori di campo intensivi con l'obiettivo esplicito di produrre raccolte comprensive ed informazioni biologiche sulle Aleocharinae, usando il vaglio e metodi di raccolta come il selezionatore di Berlese, trappole di intercettazione al volo e trappole infossate che permettono di fare paragoni tra le faune australi di aree diverse (Newton, 1985). Questi produttivi metodi di ricerca sono stati recentemente adottati adeguatamente da due musei nordamericani. Le ricerche nel Cile di Stewart e Jarmila Peck dell'Università Carleton di Ottawa e del dr. Alfred F. Newton, Jr. del Field Museum of Natural History di Chicago, hanno permesso, con l'adozione dei suddetti metodi di raccolta, di accrescere notevolmente le nostre conoscenze sulle Aleocharinae del Cile da me studiate e pubblicate (PACE, 1999, 2000, 2001). Lo statunitense dr J. S. Ashe, in occasione del congresso degli stafilinidologi a Verona, mi ha comunicato che nel suo museo di Lawrence aveva circa 
20.000 esemplari di Aleocharinae sudamericane recentemente raccolti. Me li ha offerti in studio. Gli ho esposto la mia difficoltà di presentare per pubblicazione miei lavori con stile inglese corretto, egli mi disse che potevo scrivere in italiano per la rivista del suo museo. Purtroppo nel frattempo Ashe è morto. Il lavoro per il futuro non mancherà anche per altri studiosi di Aleocharinae del Sudamerica.

\section{Materiale e Metodo}

Gli esemplari del presente lavoro appartengono alle collezioni del Museo Zoologico dell'Università Humboldt di Berlino e del DEI di Müncheberg. Altri provengono da raccolte del compianto prof. Herbert Franz, ora conservati al Naturhistorisches Museum di Vienna.

Anche lo studio tassonomico della specie sudamericane della sottofamiglia Aleocharinae, come quello d'altre regioni zoogeografiche, presenta seri problemi interpretativi che sono risolti in gran parte grazie all'esame dei caratteri dell'organo copulatore maschile e della spermateca.

Gli esemplari sono stati dissezionati con lo scopo di includere le strutture genitali in balsamo del Canadà (su piccoli rettangoli trasparenti di materiale di plastica, che accompagnano gli esemplari). Le strutture genitali sono state studiate con l'uso di un microscopio composto fino a 450 ingrandimenti e disegnate per mezzo di oculare a reticolo. L'habitus è stato da me disegnato con l'ausilio di un oculare con scala micrometrica. Tutti i disegni delle tavole sono stati da me eseguiti fino alla fase finale. Le tavole sono state da me composte al computer.

E' mia opinione, condivisa da altri entomologi, che le specie, come sono qui descritte, sono chiaramente riconoscibili in campo internazionale soprattutto dalle figure dell'edeago e della spermateca. Per questa ragione le descrizioni sono brevi e limitate. Sono descritti i caratteri incerti o non riproducibili graficamente, come il colore, la reticolazione e la granulosità. Per le specie della sottofamiglia Aleocharinae, una descrizione anche molto accurata e lunga non permette un'esatta identificazione delle varie specie. È l'osservazione dell'illustrazione dell'edeago e/o della spermateca che è di aiuto nel risolvere problemi interpretativi dati dalla sola descrizione. È omesso che il pronoto è distintamente trasverso e più largo del capo, quando questo risulta evidente dal disegno dell'habitus.

Gli olotipi delle nuove specie sono conservati nel Museo di Storia Naturale dell'Università Humboldt di Berlino (ZMHB), nel DEI di Müncheberg (DEI) e al Naturhistorisches Museum di Vienna (NMW). Paratipi si conservano negli stessi musei e in collezione dell'autore.

\section{Elenco delle specie note e descrizione dei nuovi taxa}

\section{GYROPHAENINI}

\section{Gyrophaena quassa SHARP, 1876}

Gyrophnaena quassa SHARP, 1876: 76

1 ơ, Bogota, leg. Thieme, 1877 (ZMHB).

Distribuzione: Specie nota della regione amazzonica. Facilmente riconoscibile per gli enormi occhi. 


\section{Gyrophaena nemoralis n. sp.}

(Figg. 1-3)

Materiale tipico: Holotypus ơ, Brasilien, Nova Teutonia, VI.1960, Fr. Plaumann leg. (ZMHB).

Descrizione: Lunghezza 2,7 mm. Corpo lucidissimo e rossiccio, con elitre brune tranne la base rossiccia; antenne nere con i tre antennomeri basali gialli; zampe rossicce. La punteggiatura del capo è ben visibile e rada, quella del pronoto è più evidente sul disco che ai lati dove è assente e quella delle elitre è evidente. La reticolazione del capo è superficiale, quella delle elitre è debolmente evanescente e quella dell'addome è evidente, composta di maglie isodiametriche. Il quinto urotergo libero del $\sigma^{\top}$ presenta un tubercolo mediano posteriore appiattito. Il margine posteriore del sesto urotergo libero del $\sigma^{\star}$ mostra due lobi mediani tra due spine laterali. Edeago figg. 2-3.

Comparazioni: Per la forma dell'edeago e del margine posteriore del sesto urotergo libero del maschio, la nuova specie si pone tassonomicamente vicino G. neomexicana SEevers, 1951, del Nuovo Messico. Se ne distingue per la porzione apicale dell'edeago arcuata ventralmente e non bisinuata, e per le elitre fittamente punteggiate (molto sparsamente punteggiate in neomexicana).

Etimologia: Il nome della nuova specie significa «Dei boschi», dal latino nemus = bosco.

\section{PLACUSINI}

\section{Placusa iguazuensis n. sp.}

(Figg. 4-5)

Materiale tipico: Holotypus $\odot$, Brasilien, Nova Teutonia, XI.1955, Fritz Plaumann leg. (ZMHB).

Descrizione: Lunghezza $2 \mathrm{~mm}$. Corpo debolmente lucido e nero-bruno, antenne comprese; zampe nero-brune con tibie giallo-brune. La punteggiatura del capo è superficiale. La granulosità del pronoto, delle elitre e dell'addome è evanescente. La reticolazione del capo e del pronoto è superficiale, quella delle elitre è forte e quella dell'addome è composta di maglie molto trasverse. Spermateca fig. 5.

Comparazioni: A motivo della forma della spermateca, la nuova specie mostra affinità tassonomiche con $P$. despecta Erichson, 1839, del South Carolina. Se ne distingue per il bulbo prossimale della spermateca più sviluppato e per gli occhi lunghi quanto le tempie (molto più lunghi delle tempie in despecta).

Etimologia: La nuova specie prende nome dalle famose cascate dell'Iguazù.

\section{Placusa macrocercorum n. sp.}

(Figg. 6-7)

Materiale tipico: Holotypus 우, Brasilien, Bahia (ZMHB).

Descrizione: Lunghezza 1,4 mm. Corpo lucido e bruno; antenne brune con i tre antennomeri basali bruno-rossicci; zampe posteriori giallo-rossicce, le zampe anteriori e medie sono perdute. La 
granulosità del corpo è saliente e ben visibile. La reticolazione del capo, del pronoto e dell'addome manca, quella delle elitre è visibile. Spermateca fig. 7 .

Comparazioni: Il bulbo distale della spermateca è sferico e molto sviluppato rispetto le porzioni intermedia e prossimale della stessa spermateca. I caratteri di quest'organo non si osservano nelle specie note.

Etimologia: La nuova specie prende nome dall'uccello Dicrurus macrocercus.

\section{Placusa brasiliensis n. sp.}

(Figg. 8-9)

Materiale tipico: Holotypus ㅇ, Brasilien, Rio Caraguata, $21^{\circ} 48^{\prime} \mathrm{B}, 52^{\circ} 27^{\prime} \mathrm{L}, 400 \mathrm{~m}, 1953$, Fritz Plaumann leg. (ZMHB).

Descrizione: Lunghezza 2,2 $\mathrm{mm}$. Capo e pronoto debolmente opachi, resto del corpo lucido. Corpo bruno-rossiccio con capo bruno; antenne brune con i due antennomeri basali giallorossicci; zampe gialle. La punteggiatura del capo è fittissima e superficiale, essa dà un aspetto opaco alla superficie. La granulosità del pronoto e delle elitre è fittissima ed evidente, quella dell'addome è meno fitta e altrettanto evidente. Il pronoto presenta un debole e corto solco mediano anteriore, una depressione laterale anteriore e un appiattimento mediano posteriore. Un solco mediano superficiale sta sul disco del capo. Spermateca fig. 9.

Comparazioni: La forma della spermateca della nuova specie è simile a quella di $P$. tetradon PACE, 1990, della Bolivia, tuttavia la porzione prossimale della spermateca della nuova specie ha sviluppo ridotto, mentre in tetradon la porzione prossimale della stessa spermateca è bene sviluppata.

\section{Euvira franzi n. sp.}

(Figg. 10-12)

Materiale tipico: Holotypus ơ, N Brasilien, Umg. Oeiras, Piau, H. Franz leg. (NMW).

Descrizione: Lunghezza 1,5 mm. Corpo lucido e bruno; antenne brune con i tre antennomeri basali gialli; zampe giallo-rossicce. La punteggiatura del capo è forte e assente sulla fascia longitudinale mediana. La granulosità del pronoto è fine e assai poco saliente, quella delle elitre è più forte di quella del pronoto e superficiale, quella dell'addome è ben saliente. La reticolazione del capo e del pronoto manca, quella delle elitre è superficiale e poco distinta e quella dell'addome è composta di maglie irregolari e superficiali. Gli uroterghi liberi del maschio quinto e sesto sono coperti di granuli allungati molto salienti, quasi affilati. Edeago figg. 11-12.

Comparazioni: L’edeago della nuova specie è simile a quello di E. fervidula (ERICHSON, 1839), della Colombia (serie tipica di 10 esemplari da me esaminata). Se ne distingue per l'armatura interna dell'edeago stretta (assai larga in fervidula, specialmente all'estremità distale), e per l'apice dell'edeago, in visione ventrale, diviso poco profondamente (largamente e profondamente diviso in fervidula).

Etimologia: La nuova specie è dedicata alla memoria del prof. Herbert Franz che la raccolse. 


\section{Euvira aspera n. sp.}

(Figg. 13-14)

Materiale tipico: Holotypus ㅇ, Espirito Santo, Sta. Thereze, 7.VIII.1928, O. Conde leg. (ZMHB).

Descrizione: Lunghezza 1,5 mm. Corpo debolmente lucido e giallo-bruno con capo bruno; antenne gialle sfumate di bruno dal quinto antennomero; zampe gialle. La punteggiatura del capo è profonda e fitta, quella del pronoto è composta di punti allungati nettamente impressi, quella delle elitre è superficiale. L'addome è coperto di granuli allungati che danno un aspetto di debole raspa. Il capo e il pronoto sono privi di reticolazione. La reticolazione delle elitre è superficiale. Spermateca fig. 14.

Comparazioni: La nuova specie presenta habitus simile a quello di E. fervidula (ERICHSON, 1839), della Colombia. Il capo è fortemente punteggiato nelle due specie, ma il pronoto è robustamente punteggiato nella nuova specie e debolmente in fervidula. La porzione intermedia della spermateca, inoltre, è cortissima, molto più corta dell'asse maggiore del bulbo distale della spermateca, mentre è lunga quasi quanto il diametro del bulbo distale della spermateca in fervidula.

Etimologia: Il nome della nuova specie significa «Ruvida» dal latino aspera, a motivo della forte punteggiatura del corpo.

\section{DIESTOTINI}

\section{Diestota (Apheloglossa) obsoleta (ERICHSON, 1839)}

Homalota obsoleta ERICHSON, 1839: 118

Diestota obsoleta: Bernhauer \& Scheerpeltz, 1926: 539

1 o $^{\text {e }} 2$ 우 우, Brasil, Espirito Santo, Sta. Thereza, O. Corde, 25.VII.1928 (ZMHB)

Distribuzione: Colombia. Serie tipica di $3 \sigma^{\star} o^{\star}$ e 2 ㅇ f da me esaminata.

\section{Diestota (Apheloglossa) flavipennis (ERICHSON, 1939)}

Homalota flavipennis ERICHSON, 1839: 118

Atheta (s. str.) flavipennis: Bernhauer \& SCHEerpeltz, 1926: 643

Diestota (Apheloglossa) flavipennis: PACE, 1996: 397

$2 \sigma^{\star} \sigma^{*}$, Paraguay, leg. Fieber (ZMHB).

Distribuzione: Antille e Colombia. Serie tipica da me esaminata.

\section{Diestota (Apheloglossa) laesicollis (ERICHSON, 1939)}

Homalota laesicollis ERICHSON, 1839: 109

Diestota (Apheloglossa) laesicollis: PACE, 1987: 189

$30^{*} o^{*}$ e 1 ㅇ, Brasilien, Sta. Catharina, Theresopolis, leg. Fruhstofer (ZMHB); 1 우, Rio Jan. (ZMHB); 1 , Surinam, Paramaribo, Hellas, 1908 (ZMHB).

Distribuzione: Brasile, Colombia, Guadeloupe, Perù, Bolivia. Serie tipica da me esaminata. 


\section{Diestota (Apheloglossa) melanuroides n. sp.}

(Figg. 15-17)

Atheta (Dinaraea) venezolana Bernhauer in litteris

Materiale tipico: Holotypus ơ , Carac. S., coll. Kraatz, Atheta (Dinaraea) venezolana Bernh., Ohne Kopf u. Vorder-Körper vorgefunden! Wahrscheinlich eine Folge der Präparation auf Spitzplättchen (Scheerpeltz) (DEI).

Paratypus: 1 ㅇ, senza località, leg. Moritz.

Descrizione: Lunghezza 2,5 mm. Avancorpo opaco, addome lucido. Corpo giallo-rossiccio con capo e metà posteriore delle elitre bruno-rossicci, quarto urotergo libero bruno-rossiccio; antenne brune con i due antennomeri basali bruno-rossicci e apice distale dell'undicesimo rossiccio; zampe giallo-rossicce. La punteggiatura del capo è ombelicata e superficiale. Il pronoto è privo di punteggiatura e di granulosità. La granulosità delle elitre è saliente nel maschio e poco saliente nella femmina. Una granulosità molto evidente sta sui tre uroterghi liberi basali. La reticolazione del capo e del pronoto è forte, quella delle elitre è superficiale. Il quinto urotergo libero del maschio presenta granuli allungati salienti sulla metà posteriore. Il sesto urotergo libero del maschio ha una spina a ciascun lato del margine posteriore. Edeago figg. 16-17, la spermateca dell'unico esemplare femmina è stata divorata dagli Antreni.

Comparazioni: Per la forma dell'edeago la nuova specie è simile a D. melanura (ERICHson, 1839) delle Antille (serie tipica di $10^{\top}$ e 1 o da me esaminata). Ledeago della nuova specie è profondamente arcuato al lato ventrale, mentre lo è poco in melanura. Il quinto urotergo libero del maschio, inoltre, presenta un tubercolo mediano tra due carene arcuate, mentre nella nuova specie questo carattere sessuale secondario manca.

Etimologia: Il nome della nuova specie significa "Aspetto di melanura », da Diestota melanura e

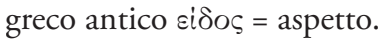

\section{Diestota (Apheloglossa) elegans n. sp.}

(Figg. 18-20)

Materiale tipico: Holotypus ơ, Umg. Pto. Natales, Südchile, H. Franz (NMW).

Descrizione: Lunghezza 2,2 $\mathrm{mm}$. Corpo debolmente lucido e rossiccio con capo e uroterghi liberi bruno-rossicci; antenne e zampe rossicce. La punteggiatura del capo è molto superficiale. La granulosità del pronoto è fine e poco evidente come quella delle elitre che è intervallata da sparsi punti evidenti. La granulosità dell'addome è fine e saliente. La reticolazione del capo è molto evidente sulla metà posteriore e molto superficiale su quella anteriore. La reticolazione del pronoto e delle elitre è forte, quella dell'addome è superficiale, tranne sul quinto urotergo libero del maschio dove è evidente e composta di maglie non trasverse, ma poligonali irregolari. Una fossetta trasversa basale profonda sta sul pronoto. La metà basale del quinto urotergo libero del maschio è priva di punteggiatura. Edeago figg. 19-20.

Comparazioni: A motivo del pronoto poco trasverso, la nuova specie si presenta simile a $D$. diffusa (Fauvel, 1901), del Messico, Colombia, Brasile, Bolivia e Perù, di cui ho esaminato due esemplari $o^{\top} o^{\star}$ del Messico determinati da Fauvel come Dinaraea diffusa Fvl. La nuova specie ha edeago 
meno robusto e non ricurvo all'apice distale come in diffusa e il margine posteriore del sesto urotergo libero del maschio è privo di sei lunghi lobi, presenti in diffusa.

Etimologia: Il nome della nuova specie significa «Elegante».

\section{Diestota (Apheloglossa) dasyporum n. sp.}

(Figg. 21-24)

Materiale tipico: Holotypus ơ, Umg. Pto. Natales, Südchile, H. Franz (NMW).

Paratypi: $2 \sigma^{\star} \sigma^{\star}$ e 1 , stessa provenienza.

Descrizione: Lunghezza $2 \mathrm{~mm}$. Corpo debolmente opaco e bruno con elitre giallo-brune con area periscutellare e laterale brune, base dell'addome e pigidio bruno-rossicci; antenne brune con i due antennomeri basali e la base del terzo giallo-rossicci; zampe giallo-rossicce. La punteggiatura del capo è fitta e superficiale. La granulosità del pronoto è molto superficiale, quella delle elitre e dell'addome è evidente. La reticolazione del capo e del pronoto è superficiale, quella delle elitre è evidente e quella dell'addome è poco visibile sui quattro uroterghi liberi basali, sul quinto è composta di maglie molto trasverse e molto superficiali. Il margine posteriore del sesto urotergo libero del maschio presenta un'incavatura mediana a ciascun lato della quale sporgono tre corti lobi della medesima lunghezza e una spina supera in lunghezza questi lobi. Edeago figg. 22-23, spermateca fig. 24.

Comparazioni: A motivo dell'habitus, la forma dell' edeago e della spermateca, la nuova specie si presenta tassonomicamente affine a D. obsoleta (ERICHSON, 1839), della Colombia, di cui ho esaminato la serie tipica di $3 o^{\star} o^{\star} \mathrm{e} 2$ 우 9 . Il pronoto della nuova specie è privo di due punti basali, presenti in obsoleta, e i lobi del margine posteriore del sesto urotergo libero del maschio sono corti nella nuova specie e lunghissimi in obsoleta. L'edeago della nuova specie è ampiamente ricurvo al lato ventrale, mentre in obsoleta lo è strettamente e la spermateca ha maggiore sviluppo di quello di obsoleta.

Etimologia: Il nome della nuova specie significa «Degli Armadilli», da Dasypus novemcinctus che è l'armadillo.

\section{Diestota (Apheloglossa) mixta n. sp.}

(Figg. 25-27)

Materiale tipico: Holotypus ${ }^{\star}$, Colombia, Muzo (ZMHB).

Descrizione: Lunghezza 1,8 mm. Corpo lucido e bruno-rossiccio con pigidio rossiccio; antenne bruno-rossicce con i tre antennomeri basali rossicci; zampe giallo-rossicce. La punteggiatura del capo è evidente e non ombelicata. La granulosità e la punteggiatura del pronoto sono assenti. La granulosità delle elitre è superficiale, quella dei tre uroterghi liberi basali è allineata in file tra loro parallele e trasverse. La reticolazione del capo è molto superficiale, quella del pronoto, delle elitre e dell'addome manca. A ciascun lato della linea mediana del quinto urotergo libero del maschio sta una debole carena. Il sesto urotergo libero del maschio presenta due deboli bozze mediane. Edeago figg. 26-27. 
Comparazioni: L'habitus della nuova specie è simile a quello di D. laesicollis (Erichson, 1839), del Brasile e Argentina, di cui ho esaminato l'holotypus ơ. Entrambe le specie presentano una fossetta mediana posteriore. Il margine posteriore del sesto urotergo libero del maschio della nuova specie presenta due larghi lobi, mentre in laesicollis i lobi sono sei e lunghissimi. Ledeago della nuova specie è stretto all'apice, in visione ventrale, mentre è largo in laesicollis.

Etimologia: Il nome della nuova specie significa «Mista», a motivo di caratteri morfologici che si trovano in varie altre specie.

\section{Eudera bisulcata (ERICHSON 1839)}

Homalota bisulcata ERICHSON, 1839: 117

Ophioglossa bisulcata: Bernhauer \& SCHEerpeltz, 1926: 569

Eudera bisulcata: PACE, 1996: 397

$1 \sigma^{\star}$, Brasil, Bahia (ZMHB); 1 ơ, Espirito Santo, Sta. Thereza, XI.1928 (ZMHB).

Distribuzione: Colombia e Brasile. Serie tipica da me esaminata.

\section{Eudera sculptilis FAUVEL, 1866}

Eudera sculptilis FAUveL, 1866: 258

1 ㅇ, Chile, Santiago, Puelmo (ZMHB).

Distribuzione: Cile. Serie tipica di $2 \sigma^{\star} o^{\star}$ e 3 ㅇ ㅇ della Cordillère de Santiago, da me esaminata.

\section{Plesiomalota (Microchara) punctulata PACE, 1983}

Microchara punctulata PACE, 1983: 304

4 es., Nova Teutonia (ZMHB).

Distribuzione: Brasile.

\section{Plesiomalota (Microchara) fragilis PACE, 1983}

Microchara fragilis PACE, 1983: 306

1 ㅇ, Nova Teutonia, VI.1960 (ZMHB).

Distribuzione: Brasile.

\section{Plesiomalota (Microchara) boliviana n. sp.}

(Figg. 41-42)

Materiale tipico: Holotypus $\odot$, Bolivien, Umg. Sta. Cruz, H. Franz leg. (NMW).

Descrizione: Lunghezza 1,6 mm. Corpo lucido e rossiccio con capo ed elitre appena oscurati di bruno, addome giallo-rossiccio; antenne brune con i due antennomeri basali e la metà basale del terzo rossicci; zampe gialle. La punteggiatura ombelicata del capo è forte e meno fitta sulla metà anteriore. La punteggiatura del pronoto è finissima, quella delle elitre è irregolare, con punti 
forti e sparsi su un fondo a punteggiatura fine. La granulosità dell'addome è fine. Una larga impressione posteriore sta sul pronoto. Spermateca fig. 42 .

Comparazioni: La spermateca della nuova specie è simile a quella di P. huascari PACE, 1983, del Perù, che presenta occhi ridotti come quelli della nuova specie. Il bulbo prossimale della spermateca, però, è molto più largo in huascari e il bulbo distale della spermateca è più sviluppato di quello della nuova specie. Le elitre sono più larghe in huascari che nella nuova specie.

\section{Plesiomalota (Microusa) belemensis n. sp.}

(Figg. 28-30)

Materiale tipico: Holotypus ơ, Brasilien, Para, Umg. Belem, H. Franz leg. (NMW).

Descrizione: Lunghezza 1,1 mm. Avancorpo debolmente lucido, addome lucido. Corpo giallorossiccio con gli uroterghi liberi terzo e quarto bruni; antenne brune con i due antennomeri basali giallo-bruni; zampe gialle. L'avancorpo è coperto di granulosità fitta e saliente che dà un aspetto rugoso alla superficie. L'avancorpo è privo di reticolazione. La reticolazione dell'addome è superficiale. Il pronoto presenta due brevi e superficiali depressioni mediane posteriori. Edeago figg. 29-30.

Comparazioni: La nuova specie per la forma del suo edeago è simile a P. tenella PACE, 1987, di Guadaloupe. Se ne distingue per gli occhi molto ridotti (lunghi quanto le tempie in tenella) e per l'edeago profondamente ricurvo al lato ventrale (poco ricurvo in tenella).

Etimologia: Il nome della nuova specie significa «Di Belem», sua località tipica.

\section{Plesiomalota (Microusa) minima n. sp.}

(Figg. 31-32)

Materiale tipico: Holotypus ㅇ, Brasilien, Rio Grande do Sul, Umg. Sta. Maria, H. Franz leg. (NMW).

Paratypus: 1 , stessa provenienza.

Descrizione: Lunghezza 1,27 mm. Avancorpo debolmente lucido, addome lucido. Corpo giallo con elitre giallo-brune; antenne giallo-brune con i tre antennomeri basali gialli; zampe gialle. La punteggiatura del capo è ombelicata, fitta, evidente, ma assente sulla fronte che è coperta di reticolazione superficiale. La granulosità del pronoto è confusa nella reticolazione. La granulosità delle elitre e dell'addome è superficiale. La reticolazione delle elitre è evidente, quella dell'addome è molto superficiale. Spermateca fig. 32 .

Comparazioni: La spermateca della nuova specie è avvolta in spire come quella di $P$. punctulata PACE, 1983, del Brasile. Le spire della spermateca della nuova specie, tuttavia, sono nettamente più numerose, circa 10, mentre in punctulata la spermateca ne ha 3.

Etimologia: Il nome della nuova specie significa «Molto piccola», a motivo della lunghezza del corpo. 


\section{Plesiomalota (Microusa) surinamensis n. sp.}

(Figg. 43-44)

Materiale tipico: Holotypus ㅇ, Surinam, Bergendal, 1908, C. Heller leg. (ZMHB).

Paratypi: 2 우, stessa provenienza.

Descrizione: Lunghezza 1,7 mm. Corpo lucido e giallo-rossiccio con capo rossiccio ed elitre bruno-rossicce; antenne rossicce con i due antennomeri basali giallo-rossicci; zampe gialle. La punteggiatura del capo è forte e fittissima, assente sulla fronte. Il pronoto non mostra punteggiatura o granulosità. La granulosità delle elitre è molto superficiale, quella dell'addome è fine. La reticolazione del capo e dei tre uroterghi liberi basali è superficiale, quella del pronoto, delle elitre e dei restanti uroterghi liberi è evidente. Un largo appiattimento mediano sta sul pronoto. Spermateca. fig. 44.

Comparazioni: Il bulbo prossimale della spermateca è sviluppato in modo enorme nella nuova specie. Nessuna specie nota presenta questo singolare carattere.

\section{Plesiomalota (Plesiomalota) tarapotensis n. sp.}

(Figg. 33-36)

Materiale tipico: Holotypus ơ, Peru, Umg. Tarapoto, H. Franz leg. (NMW).

Paratypi: 7 es., stessa provenienza.

Descrizione: Lunghezza 1,7 $\mathrm{mm}$. Capo e pronoto opachi, elitre debolmente lucide e addome lucido. Corpo rossiccio con elitre oscurate di bruno sul disco; antenne bruno-rossicce con antennomero basale e tre quarti apicali dell'undicesimo giallo-rossicci; zampe giallo-rossicce. La punteggiatura del capo e del pronoto è fittissima tanto da dare un aspetto opaco alla superficie. Le elitre sono coperte di punteggiatura fitta e di reticolazione evidente. L'addome è privo di reticolazione. Edeago. figg. 34-35, spermateca fig. 36.

Comparazioni: La nuova specie è distinta da P. roedingeri (Bernhauer, 1941), del Perù, di cui ho esaminato l'holotypus $\sigma^{*}$, per l'edeago bruscamente incavato al lato ventrale (bisinuoso in roedingeri) e molto più largo alla porzione apicale distale, in visione ventrale.

Etimologia: Il nome della nuova specie significa «Di Tarapoto». Tarapoto è la sua località tipica.

\section{Plesiomalota (Plesiomalota) iguazuensis n. sp.}

(Figg. 45-47)

Materiale tipico: Holotypus ơ , Brasilien, Nova Teutonia, XI.1955, Plaumann, leg. (ZMHB).

Paratypi: 2 ํํ o e 1 우 , Brasilien, Nova Teutonia, VI.1960, Plaumann leg.

Descrizione: Lunghezza 2,4 $\mathrm{mm}$. Corpo con riflessi argentei, forse a causa di preparazione per l'esame al microscopio a scansione. Corpo rossiccio con capo bruno, elitre brune con base rossiccia, quarto urotergo libero bruno; antenne brune con i due antennomeri basali e l'undicesimo bruno-rossicci; zampe rossicce. La punteggiatura del capo è ombelicata, fitta e forte, quella del pronoto è assente, 
quella delle elitre è composta di punti allungati evidenti. La granulosità dell'addome è superficiale. La reticolazione del capo è ben visibile, quella del pronoto è forte, quella delle elitre e dell'addome è superficiale, nel fondo dei solchi trasversi basali è forte e composta di maglie molto ampie e sul quinto urotergo libero del maschio a maglie molto trasverse e superficiali. Il pronoto presenta un debole solco mediano posteriore a forma di $\mathrm{U}$ e un corto solco mediano anteriore. Il sesto urotergo libero del maschio mostra un rilievo mediano triangolare appiattito. Edeago figg. 46-47.

Comparazioni: La nuova specie presenta il pronoto bisolcato come in P. fasciatipennis (FAIRMAIRE \& Germain, 1861), di cui ho esaminato la serie tipica di 1 o e 2 ㅇ ㅇ․ . Ma il sesto urotergo libero del maschio della nuova specie presenta un rilievo mediano triangolare, mentre in fasciatipennis tale carattere manca, ma il margine posteriore del sesto urotergo libero del maschio è dentellato. L'edeago è simile nelle due specie, ma nella nuova i lati della porzione apicale distale, in visione ventrale, sono lobati, mentre non lo sono in fasciatipennis.

Etimologia: La nuova specie prende nome dalle famose cascate dell'Iguazù.

\section{Plesiomalota (Plesiomalota) bradyporum n. sp.}

(Figg. 37-40)

Materiale tipico: Holotypus ơ , Brasilien, Bahia (ZMHB).

Paratypi: 7 es., stessa provenienza.

Descrizione: Lunghezza 1,8 mm. Avancorpo opaco, addome lucido. Corpo bruno con elitre giallo-brune; antenne brune con i due antennomeri basali giallo-rossicci; zampe gialle. La punteggiatura del capo è ombelicata, fitta e forte, quella del pronoto è poco visibile, quella delle elitre è fine e fitta. La granulosità dell'addome è evidente e fitta, diradata solo sul quinto urotergo libero. La reticolazione del capo è vigorosa, quella del pronoto è ben visibile, quella delle elitre e dell'addome manca. Sul pronoto si trovano due deboli solchi mediani posteriori. Edeago figg. 38-39, spermateca fig. 40.

Comparazioni: A motivo dell'edeago profondamente arcuato al lato ventrale, la nuova specie è probabilmente tassonomicamente affine a P. hispidula (Fauvel, 1866), del Cile, di cui ho esaminato la serie tipica di 1 o e 3 우. Ma in visione ventrale l'edeago della nuova specie è stretto, mentre è larghissimo in hispidula.

Etimologia: Il nome della nuova specie significa «Dei Bradipi», da Bradypus tridactylus famoso mammifero arboricolo sudamericano.

\section{HOMALOTINI}

\section{Tropocoenonica n. gen.}

(Figg. 48-54)

Diagnosi: A motivo della forma della spermateca, non avvolta in numerose spire, il nuovo genere mostra maggiore affinità al genere Coenonica KrAATZ, 1857, diffuso in Asia e Africa, che al genere Diestota Mulsant \& Rey, 1870, presente anche in Sudamerica. Il nuovo genere è differente da Coenonica per la ligula divisa solo all'estremità apicale, fig. 51 e non fino oltre la sua metà, fig. 64, 
per la presenza di un'evidente carena mediana del mesosterno, assente in Coenonica e per la forma della spermateca.

Descrizione: Habitus simile a quello di Diestota, con pronoto solcato ad U posteriormente come le specie di questo genere; tempie marginate, palpi labiali di 2 articoli, fig. 51; ligula lunga e divisa all'apice distale, fig. 51; paraglosse sporgenti in avanti; mento trapezoidale poco incavato al margine anteriore, fig. 54; palpi mascellari di 4 articoli, fig. 53; processo mesosternale acuto e carenato sulla linea mediana; mesocoxe contigue; formula tarsale 4-4-5; primo mesotarsomero lungo quanto il seguente; primo metatarsomero lungo quanto i 2 seguenti riuniti; edeago figg. 49-50; spermateca fig. 52 .

Typus generis: Tropocoenonica catharinensis n. sp.

Etimologia: Il nome del nuovo genere significa "Coenonica carenata», da Coenonica, genere di Aleocharinae e $\tau \sigma o \dot{\tau} \iota \varsigma$ = carena in greco antico. La carena è quella mediana del mesosterno.

\section{Tropocoenonica catharinensis $\mathrm{n} . \mathrm{sp}$.}

(Figg. 48-54)

Materiale tipico: Holotypus o, Brasilien, Nova Teutonia, 300-500 m, VI-1960, leg. F. Plaumann (ZMHB).

Paratypi: 1 ot e 3 우 오, Brasilien, Nova Teutonia, XV.1955, leg. F. Plaumann.

Descrizione: Lunghezza 2,7 $\mathrm{mm}$. Corpo lucido e rossiccio; antenne rossicce con i tre antennomeri basali e l'undicesimo giallo-rossicci; zampe rossicce. La granulosità del capo è molto superficiale, quella del pronoto e delle elitre è saliente. La reticolazione del capo, delle elitre e dell'addome è molto superficiale, quella del pronoto manca. Questo presenta un'impressione posteriore mediana ad U. Il quinto urotergo libero del maschio è coperto di reticolazione forte e carene e tubercoli distribuiti come da fig. 48. Il margine posteriore del sesto urotergo libero del maschio presenta cinque lunghi lobi tra due spine laterali. Edeago figg. 49-50, spermateca fig. 52.

Etimologia: Il nome della nuova specie significa «Di Caterina», dallo stato brasiliano di Santa Caterina.

\section{BOLITOCHARINI}

\section{Prosoponotha brasiliensis n. sp.}

(Figg. 55-58)

Materiale tipico: Holotypus ơ, Nova Teutonia, VII.1959, leg. Plaumann (ZMHB).

Descrizione: Lunghezza 1,8 mm. Corpo lucido e giallo-rossiccio; antenne brune con i tre antennomeri basali e l'undicesimo giallo-rossicci; zampe giallo-rossicce. Il capo è privo di punteggiatura, granulosità e reticolazione. La granulosità del pronoto e delle elitre è molto superficiale, quella dell'addome è saliente solo lungo il margine posteriore di ciascun urotergo libero. La reticolazione del pronoto manca, quella delle elitre è superficiale e quella dell'addome è evidente. Il quinto urotergo libero del maschio ha tre tubercoli allungati distribuiti come da 
fig. 55. Il margine posteriore del sesto urotergo libero del maschio ha sei lobi di modesta lunghezza tra due corte spine laterali. Edeago figg. 56-57, labio con palpo mascellare fig. 58.

Comparazioni: Per la forma dell'edeago, la nuova specie è simile a $P$. osornensis PACE, 2000, del Cile, ma nella nuova specie l'apice distale dell'edeago è strettissimo (di media larghezza in osornensis) e l'armatura genitale interna dell'edeago è lunghissima e robusta nella nuova specie, mentre è corta ed esile in osornensis.

\section{Dispachyglossa n. gen.}

(Figg. 59-63)

Diagnosi: Il nuovo genere è affine al genere Bolitochara MANNERHEIM, 1830, perché presenta tre articoli dei palpi labiali, il mesosterno carenato e spermateca di forma simile. Se ne distingue per la ligula più lunga e soprattutto per essere fortemente sclerificata (membranosa in Bolitochara) tanto da avere colore bruno. Ciò indica che è occupata probabilmente una nicchia ecologica differente da quella di Bolitochara.

Descrizione: Habitus simile a quello di Bolitochara; tempie non marginate, palpi labiali di 3 articoli; ligula fortemente sclerificata e bruna, divisa all'apice fig. 61; paraglosse sporgenti in avanti; mento trapezoidale con margine anteriore arcuato all'indietro, fig. 63; palpi mascellari di 4 articoli, fig. 62; processo mesosternale acuto e carenato; mesocoxe contigue; formula tarsale 44-5; primo metatarsomero lungo quanto i 2 tarsomeri seguenti riuniti; spermateca fig. 60.

Typus generis: Dispachyglossa elegans n. sp.

Etimologia: Il nome del nuovo genere significa «Lingua due volte spessa», dal greco antico $\delta i \varsigma=$ due volte, $\pi \alpha \chi \dot{\nu} \varsigma=$ spessa e $\gamma \lambda \dot{\omega} \sigma \sigma \alpha=$ lingua.

\section{Dispachyglossa elegans n. sp.}

(Figg. 59-63)

Materiale tipico: Holotypus o , località illeggibile: Doh zons Lumileta (ZMHB).

Paratypus: 1 ㅇ, stessa provenienza.

Descrizione: Lunghezza $4 \mathrm{~mm}$. Corpo lucidissimo e giallo-rossiccio con capo e quarto urotergo libero bruno-rossicci; antenne bruno-rossicce con i tre antennomeri basali rossicci; zampe giallorossicce. La punteggiatura del capo è doppia, composta di punti fini e di punti forti assai ben visibili. La punteggiatura del pronoto è superficiale, quella delle elitre è forte. Una evidente granulosità copre l'addome, tranne nel fondo dei solchi trasversi basali degli uroterghi che sono nettamente punteggiati. Il corpo è privo di reticolazione, tranne che sul sesto urotergo libero del maschio dove è molto trasversa e superficiale. Il pronoto presenta una debole fossetta mediana posteriore. Spermateca fig. 60.

Etimologia: Il nome della nuova specie significa «Elegante». 


\section{Thecturota maculata n. sp.}

(Figg. 65-66)

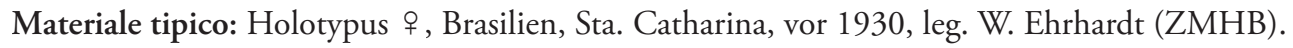

Descrizione: Lunghezza 1,6 mm. Corpo lucido e giallo con capo rossiccio, elitre bruno-rossicce con base giallo-bruna, quarto urotergo libero con una gran macchia bruna mediana; antenne bruno-rossicce con i due antennomeri basali e l'undicesimo giallo-rossicci; zampe gialle. La punteggiatura del capo è superficiale e rada, quella delle elitre è ben visibile. La granulosità del pronoto e dell'addome è fine e poco evidente. La reticolazione del capo è molto superficiale, quella del pronoto è evidente, quella delle elitre è superficiale e quella dell'addome manca. Il quinto urotergo libero della femmina ha un'impressione basale profonda coperta di punteggiatura forte. Spermateca fig. 66.

Comparazioni: La nuova specie è distinta da T. cordillerana (Bernhauer, 1921), dell'Argentina, di cui ho esaminato l'holotypus + , oltre che per la presenza di una macchia bruna dell'addome (assente in cordillerana), per la forma della spermateca. Questa nella nuova specie ha la porzione prossimale stretta, mentre è larga in cordillerana.

Etimologia: Il nome della nuova specie significa "Macchiata», a motivo della macchia bruna dell'addome.

\section{Habramicrada n. gen.}

(Figg. 67-71)

Diagnosi: Habitus e spermateca simili a quelli di Paroxypodinus Cameron, 1933, del Borneo, ma palpi labiali di tre articoli (quattro in Paroxypodinus) e ligula intera e stretta (larga e divisa all'estremità apicale in Paroxypodinus).

Descrizione: Corpo minutissimo da 1,07 a 1,35 mm e poco convesso; pronoto più ristretto in avanti che all'indietro, addome a lati paralleli; tempie fortemente marginate; palpi labiali di 3 articoli di cui il secondo assai breve, fig. 69; ligula intera e stretta fig. 69, paraglosse sporgenti in avanti; mento trapezoidale con margine anteriore debolmente arcuato, fig. 71; palpi mascellari di 4 articoli, fig. 70; processo mesosternale acuto e non carenato; mesocoxe contigue; formula tarsale 4-4-5; primo metatarsomero lungo quanto il seguente; spermateca con porzione prossimale avvolta in spire, figg. 68, 75, 80 .

Typus generis: Habramicrada boliviensis n. sp.

Etimologia: Il nome del nuovo genere significa «Piccolissimo fastidio grazioso» dal greco antico $\dot{\alpha} \beta \varrho o \dot{ } \varsigma$ grazioso, $\mu \varkappa \varrho o \dot{\varsigma}=$ piccolo e $\dot{\alpha} \delta \circ \varsigma=$ fastidio. Il fastidio deriva dalla difficoltà nel dissezionare esemplari di taglia piccolissima come quelli delle specie attribuite a questo genere.

\section{Habramicrada boliviensis n. sp.}

(Figg. 67-71)

Materiale tipico: Holotypus 우 Bolivien, Sta. Cruz, leg. H. Franz (NMW).

Descrizione: Lunghezza 1,16 mm. Corpo lucido e giallo-rossiccio chiaro con tre quarti posteriori delle elitre e quarto urotergo libero bruni; antenne brune con i due antennomeri basali e 
l'undicesimo giallo-rossicci; zampe gialle. La punteggiatura del capo è fine. La granulosità del pronoto e delle elitre è superficiale. La reticolazione del capo e del pronoto manca, quella delle elitre è ben visibile. Spermateca fig. 68 .

Comparazioni: La nuova specie si distingue dalle altre specie essenzialmente per la forma della spermateca che presenta la porzione prossimale avvolta in una spira e mezzo.

\section{Habramicrada anularis n. sp.}

(Figg. 72-75)

Materiale tipico: Holotypus ơ, Brasilien, Para, Umg. Belem, leg. H. Franz (NMW).

Paratypus: 1 , stessa provenienza.

Materiale tipico: Lunghezza 1,1 mm. Corpo lucido e giallo-rossiccio chiaro con elitre, tranne agli omeri, e quarto urotergo libero bruni; antenne brune con i tre antennomeri basali e i tre terminali gialli; zampe gialle. La punteggiatura del capo è ben visibile, quella del pronoto è superficiale. La granulosità delle elitre è evanescente e quella dell'addome è evidente. Il corpo è privo di reticolazione tranne sulle elitre dove la reticolazione è superficiale. Edeago figg. 73-74, spermateca fig. 75.

Comparazioni: La nuova specie si distingue dalle altre specie qui descritte essenzialmente per la forma della spermateca che presenta la porzione prossimale avvolta in numerose spire aggrovigliate.

Etimologia: Il nome della nuova specie significa «Anulare», dalla fascia o anello bruno dell'addome.

\section{Habramicrada peruviana n. sp.}

(Figg. 76-78)

Materiale tipico: Holotypus ơ, Peru, Umg. Tarapoto, leg. H. Franz (NMW).

Descrizione: Lunghezza $1,35 \mathrm{~mm}$. Corpo lucido e giallo-rossiccio con elitre e addome brunorossicci; antenne bruno-rossicce con i quattro antennomeri basali giallo-rossicci; zampe giallorossicce con femori oscurati di bruno. La punteggiatura del capo è ben visibile, quella del pronoto manca. La granulosità delle elitre è fine, quella dell'addome è poco saliente. Il corpo è privo di reticolazione, tranne sulle elitre dove è ben visibile. Edeago figg. 77-78.

Comparazioni: A motivo della taglia corporea maggiore e per la forma dell'edeago, la nuova specie è distinta dalle altre specie qui descritte.

Etimologia: Il nome della nuova specie significa «Del Perù».

\section{Habramicrada incaica n. sp.}

(Figg. 79-80)

Materiale tipico: Holotypus + , Peru, Umg. Tarapoto, leg. H. Franz (NMW).

Descrizione: Lunghezza 1,07 mm. Corpo lucido e giallo-rossiccio con metà posteriore delle elitre e quarto urotergo libero giallo-bruni; antenne brune con i due antennomeri basali e l'undicesimo 
gialli; zampe gialle. La punteggiatura del capo è superficiale, quella del pronoto è assai superficiale. La granulosità delle elitre è poco visibile. Una reticolazione superficiale si trova solo sulle elitre, sul resto del corpo la reticolazione manca.

Comparazioni: La nuova specie presenta una minuscola spermateca con porzione prossimale avvolta in una spira e mezzo e una taglia corporea ridottissima. Per questi caratteri è distinta dalle specie qui descritte.

Etimologia: Il nome della nuova specie significa «Degli Incas», antico popolo del Perù.

\section{PAGLINI}

\section{Pagla (Parallelopagla) n. subgen.}

Il nuovo sottogenere è qui proposto per il fatto che tutte le specie del genere Pagla BLACKwelder, 1952, finora note, presentano corpo ristretto in avanti e all'indietro come per gran parte delle Oxypoda, mentre non si osserva questo carattere nella specie tipo del sottogenere: Pagla levis n. sp. Non è genere a sé stante perché ligula, palpi labiali, formula tarsale e forma della spermateca rientrano nell'ambito del genere Pagla. Il nuovo sottogenere si distingue per i caratteri dati nella seguente chiave:

1. Corpo ristretto in avanti e all'indietro come in Oxypoda .......................... Pagla (Pagla)

- Corpo a lati paralleli Pagla (Parallelopagla n. subg.)

Typus subgeneris: Pagla (Parallelopagla) levis n. sp.

Etimologia: Dal greco antico $\pi \alpha \varrho \alpha \lambda \lambda \eta \lambda \circ \varsigma=$ parallelo e Pagla .

\section{Pagla (Parallelopagla) levis n. sp.}

(Figg. 81-83)

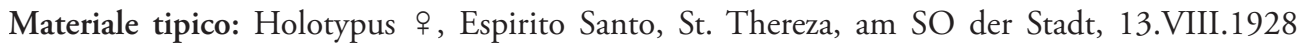
(ZMHB).

Paratypi: 6 우 옹 stessa provenienza.

Descrizione: Lunghezza 2,5 mm. Corpo lucido e bruno-rossiccio con pigidio giallo-rossiccio; antenne bruno-rossicce con i tre antennomeri basali giallo-rossicci; zampe bruno-rossicce con ginocchia, estremità distale delle tibie e tarsi rossicci. La punteggiatura del capo è superficiale, quella del pronoto è ben visibile e quella delle elitre è superficiale. Solo sul capo è presente una reticolazione che è superficiale, sul resto del corpo la reticolazione manca. Spermateca fig. 82, labio con palpo labiale fig. 83 .

Comparazioni: La forma del corpo, a lati paralleli, distingue la nuova specie dalle specie note che presentano corpo ristretto in avanti e all'indietro.

Etimologia: Il nome della nuova specie significa «Leggera», a motivo della punteggiatura del corpo poco profonda. 


\section{FALAGRIINI}

\section{Falagria (Leptagria) boliviana n. sp.}

(Figg. 84-85)

Materiale tipico: Holotypus ㅇ, Bolivien, Villa Montès, 17.XI.1930, leg. Dr. Eisentraut (ZMHB).

Descrizione: Lunghezza 2,8 $\mathrm{mm}$. Corpo lucido e bruno con elitre e i tre uroterghi liberi basali giallo-bruni; antenne e zampe rossicce. La punteggiatura del capo è fine e superficiale, sulla fronte però si trova una distinta granulosità. La granulosità del pronoto è visibile solo sulla metà anteriore, il resto della superficie è coperto di punteggiatura fine. Lo scutello è coperto di granuli salienti. La punteggiatura delle elitre è fine. La granulosità dell'addome è ben visibile. Il fondo dei solchi trasversi basali degli uroterghi liberi basali è chiaramente punteggiato. Il corpo è privo di reticolazione, tranne nel fondo dei solchi trasversi basali dell'addome dove è forte. Il solco mediano del pronoto è profondo, ma non raggiunge il margine anteriore. Spermateca fig. 85 .

Comparazioni: A motivo della forma della spermateca, la nuova specie è tassonomicamente affine a F. fissula Erichson, 1839, del Venezuela e Brasile, di cui ho esaminato l'holotypus o e un esemplare + . Se ne distingue per avere la spermateca robusta (esile in fissula), con introflessione apicale del bulbo distale della spermateca presente (assente in fissula). Le antenne di fissula sono bruno-rossicce con i due antennomeri basali e l'undicesimo gialli, mentre nella nuova specie le antenne sono uniformemente rossicce.

\section{Meronera tersa n. sp.}

(Figg. 86-88)

Materiale tipico: Holotypus $\sigma^{\star}$, Bolivien, Umg. Sta. Cruz, leg. H. Franz (NMW).

Descrizione: Lunghezza 2,3 $\mathrm{mm}$. Corpo lucido e bruno con capo bruno-rossiccio e con i due uroterghi liberi basali gialli con base bruna; antenne bruno-rossicce con i due antennomeri basali giallo-rossicci, zampe brune con protibie, estremità distale e basale delle mesotibie e delle metatibie e tutti i tarsi giallo-rossicci. La punteggiatura del capo è poco distinta, quella del pronoto è fine e superficiale, quella delle elitre è ben visibile. L'addome è privo sia di punteggiatura che di granulosità, tranne sui due terzi posteriori del quinto urotergo libero del maschio coperti di granulosità e setoline. La reticolazione del capo è molto superficiale, quella del resto della superficie manca. Sul pronoto si trovano alcuni forti punti isolati. Edeago figg. 87-88.

Comparazioni: Il corpo della nuova specie è molto simile a quello di $M$. venustula (ERICHSON, 1839), del Nordamerica, di cui ho esaminato la serie tipica di 1 o e 4 ㅇ 우. Anche la reticolazione del capo e del pronoto e il colore del corpo sono simili, tranne per il colore delle zampe che in venustula sono gialle. Le due specie si distinguono soprattutto per la forma dell'edeago: quello di venustula presenta lati convergenti verso l'apice distale, in visione ventrale, mentre nella nuova specie sono pressoché paralleli. L'armatura genitale interna dell'edeago di venustula, inoltre, presenta due lamine falciformi, assenti nell'edeago della nuova specie, sostituite da sclerificazioni di forma molto differente.

Etimologia: Il nome della nuova specie significa «Nitida», per l'addome quasi senza punteggiatura. 


\section{Ringraziamenti}

Per avermi affidato in studio il materiale oggetto del presente lavoro rivolgo i miei più cordiali ringraziamenti al Dr. Manfred Uhlig del Museo Zoologico dell'Università Humboldt di Berlino e al Dr. Lothar Zerche del DEI di Müncheberg. Per il prestito di tipi ringrazio il Dr. A.F. Newton jr., del Field Museum of Natural History di Chicago e ancora il Dr. Manfred Uhlig e il Dr. Lothar Zerche.

\section{Bibliografia}

Arnoldi, L. V.; Zherichin, V. V.; Nikritkin, L. M. \& Ponomarenko, A. G. 1977: Coleotteri Mesozoici [in russo]. - Transactions of the Paleontological Institute 161: 1-204.

Bernhauer, M. 1941: Staphylinidae (Coleoptera). - Beiträge zur Fauna Perous 1: 277-293.

Bernhauer, M. \& Scheerpeltz, O. 1926: Coleopterorum Catalogus, Berlin pars 82, Staphylinidae 6: 499-988.

BLACKWELDER, R. E. 1952: The generic names of the Beetle family Staphylinidae. - Smithsonian Institution, United States National Museum Bulletin 200: 483 pp.

Cameron, M. 1933: Staphylinidae (Coleoptera) from Mount Kinabalu. - Journal of Federal Malay State Museum 17: 338-360.

Erichson, W. K. 1839: Genera et species Staphylinorum Insectorum Coleopterorum familiae, Berlin: F. H. Morin. 1: 1-400.

Fairmaire, M. L. \& Germain, P. 1861: Révision dés Coléoptères du Chili. - Annales de la Société Entomologique de France 1: 405-456.

Fauvel A. 1866: Faune de Chili. Insectes Coléoptères Staphylinides. - Bulletin de la Société Linneénne de Normandie, 10: 250-353.

Fauvel A. 1901: Voyage de M. le Dr. Ed. Bugnion au Venezuela, en Colombie et aux Antilles. - Revue d'Entomologie 20: 69-91.

Herman, L. H. 2001: Catalog of the Staphylinidae (Insecta: Coleoptera). 1758 to the end of the second millennium. I. Introduction, History, Biographical sketches, and Omaliine Group. - Bulletin of the American Museum of Natural History, New York 265: 1-649.

KraAtz, G. 1857: Genera Aleocharinorum Illustrata. - Linnaea Entomologica 11: 1-43.

Lawrence, J. F. \& Newton, A. F. Jr. 1982: Evolution and classification of beetles. - Annual Review of Ecology and Systematics 13: 261-290.

Mannerheim, C. G. 1830: Précis d'un nouvel arrangement de la Famille des Brachélytres de l'Ordre des Insectes Coléoptères. - Mémoires de l'Académie Impériale des Sciences de St. Pétersbourg 1: 415-511.

Mulsant, E. \& Rey, Cl. 1870: Description d'un genre nouveau de l'ordre des Coléoptères. - Opuscules entomologiques, quatorzième cahier: 194-199.

Newton, A. F. JR. 1985: South temperate Staphylinoidea (Coleoptera): their potential for biogeographic analysis of austral disjunctions. - In: G. E. BALL (ed.). Taxonomy, Phylogeny and Zoogeography of Beetles and Ants: 180-220. - Dr W. Junk Publishers, Dordrecht.

PACE, R. 1983: Nuove Aleocharinae brasiliane e boliviane del Museo Zoologico dell'Università Humboldt di Berlino (Coleoptera, Staphylinidae) (XXXVIII tris Contributo alla conoscenza delle Aleocharinae).

- Giornale italiano d'Entomologia 2: 295-316, 92 figg.

PACE, R. 1986: Aleocharinae del Perù (Coleoptera Staphylinidae) (LXXXV Contributo alla conoscenza delle Aleocharinae). - Redia 69: 417-467, 23 figg.

PACE, R. 1987: Aleocharinae dell'isola della Guadalupa (Antille) (Coleoptera, Staphylinidae) (LXXXIX Contributo alla conoscenza delle Aleocharinae). - Folia Entomologica Hungarica, Budapest 48: 187-205, 108 figg. 
PACE, R. 1990: Aleocharinae neotropiche del Museo Ungherese di Storia naturale (Coleoptera, Staphylinidae) (XC Contributo alla conoscenza delle Aleocharinae). - Annales Historico-Naturales Musei Nationalis Hungarici, Budapest, Pars Zoologica 81: 53-107, 264 figg.

PaCE, R. 1996: Aleocharinae della Colombia e dell'Ecuador, Parte I (Coleoptera, Staphylinidae) (131 Contributo alla conoscenza delle Aleocharinae). - Revue suisse de Zoologie 103: 395-437, 155 figg.

PACE, R. 1999: Aleocharinae del Cile (Coleoptera, Staphylinidae) (114 Contributo alla conoscenza delle Aleocharinae). - Bollettino del Museo civico di Storia naturale di Verona 23: 119-210.

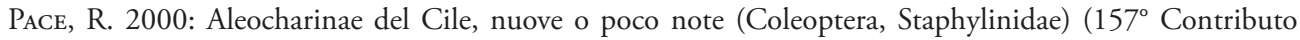
alla conoscenza delle Aleocharinae). - Bollettino del Museo regionale di Scienze naturali di Torino 17: 335-510.

Pace, R. 2001: Nuove specie cilene del genere Leptusa. Monografia del genere Leptusa KraATz: Supplemento XII. - Lavori della Società Veneziana di Scienze Naturali di Venezia 26: 7-14.

Seevers, C. H. 1951: A Revision of the North American and European Staphylinid Beetles of the Subtribe Gyrophaenae (Aleocharinae, Bolitocharini). - Fieldiana Zoology 32: 660-762.

Sharp, D. 1876: Contribution to an insectfauna of the Amazon Valley, Staphylinidae. - Transactions of the Entomological Society of London 1: 27-424.

Tiкномirova, A. L. 1968: Jurassic Staphilinids of the Karatau (Coleoptera, Staphylinidae). - In: Insetti Giurassici del Karatau [in russo] Nauka Publishing House, Moscow: 139-154. 

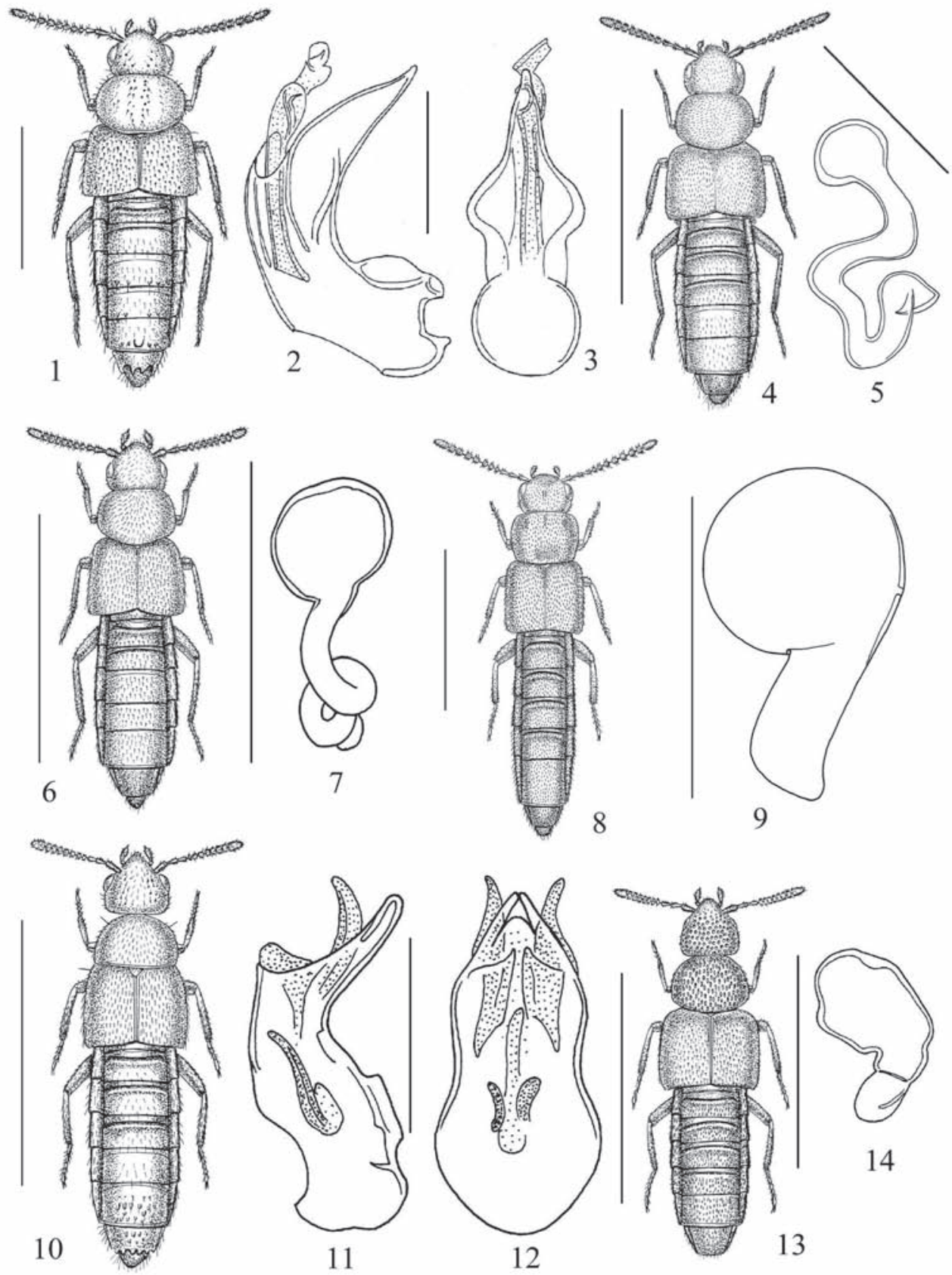

Figg. 1-14: Habitus, edeago in visione laterale e ventrale e spermateca. 1-3. Gyrophaena nemoralis n. sp.; 4-5. Placusa iguazuensis n. sp.; 6-7. Placusa macrocercorum n. sp.; 8-9. Placusa brasiliensis n. sp.; 10-12. Euvira franzi n. sp.; 13-14. Euvira aspera n. sp. Habitus scala $1 \mathrm{~mm}$, altre scale $0,1 \mathrm{~mm}$. 


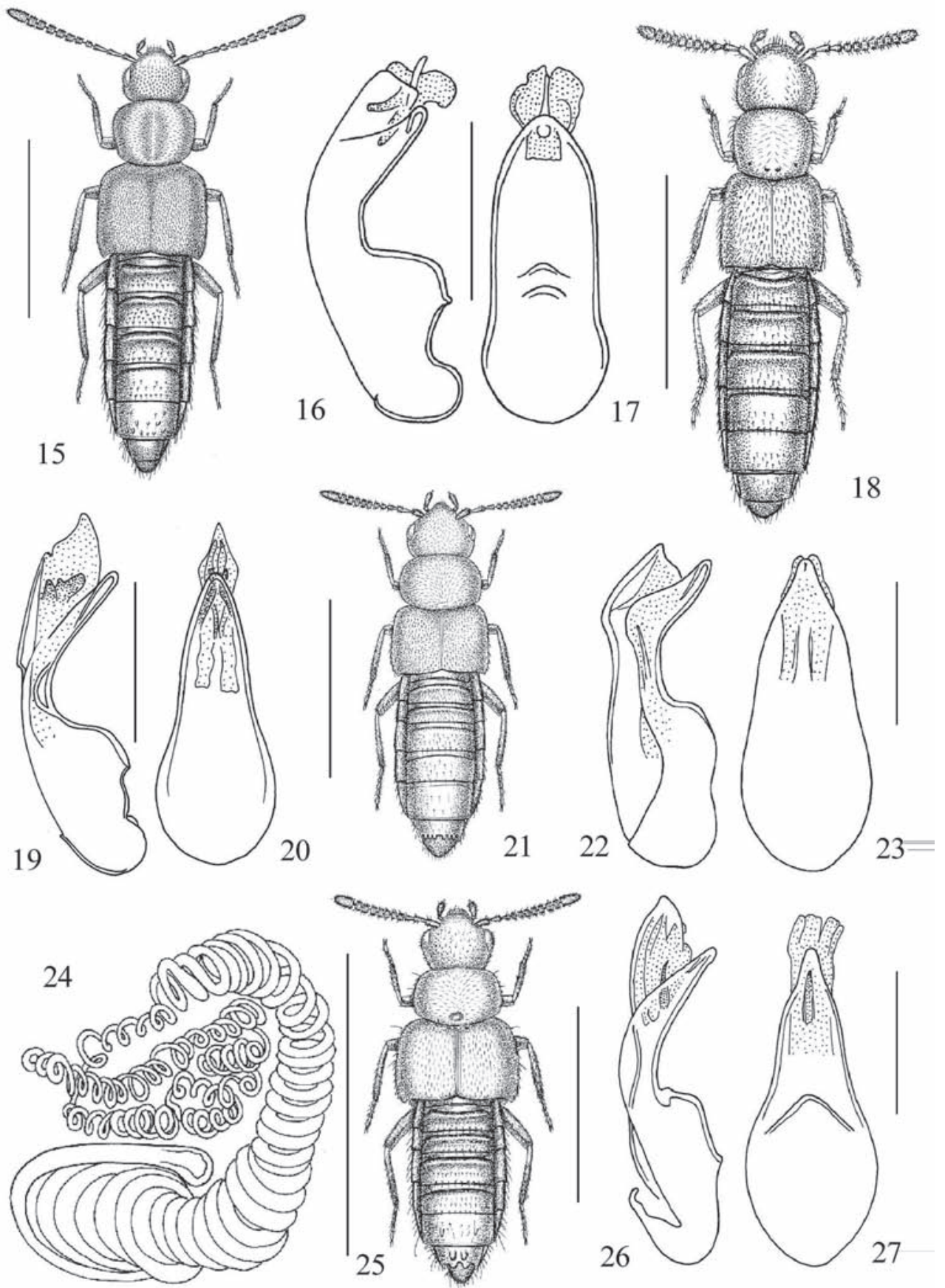

Figg. 15-27: Habitus, edeago in visione laterale e ventrale e spermateca. 15-17. Diestota (Apheloglossa) melanuroides n. sp.; 18-20. Diestota (Apheloglossa) elegans n. sp.; 21-24. Diestota (Apheloglossa) dasyporum n. sp.; 25-27. Diestota (Apheloglossa) mixta n. sp. Habitus scala $1 \mathrm{~mm}$, altre scale $0,1 \mathrm{~mm}$. 

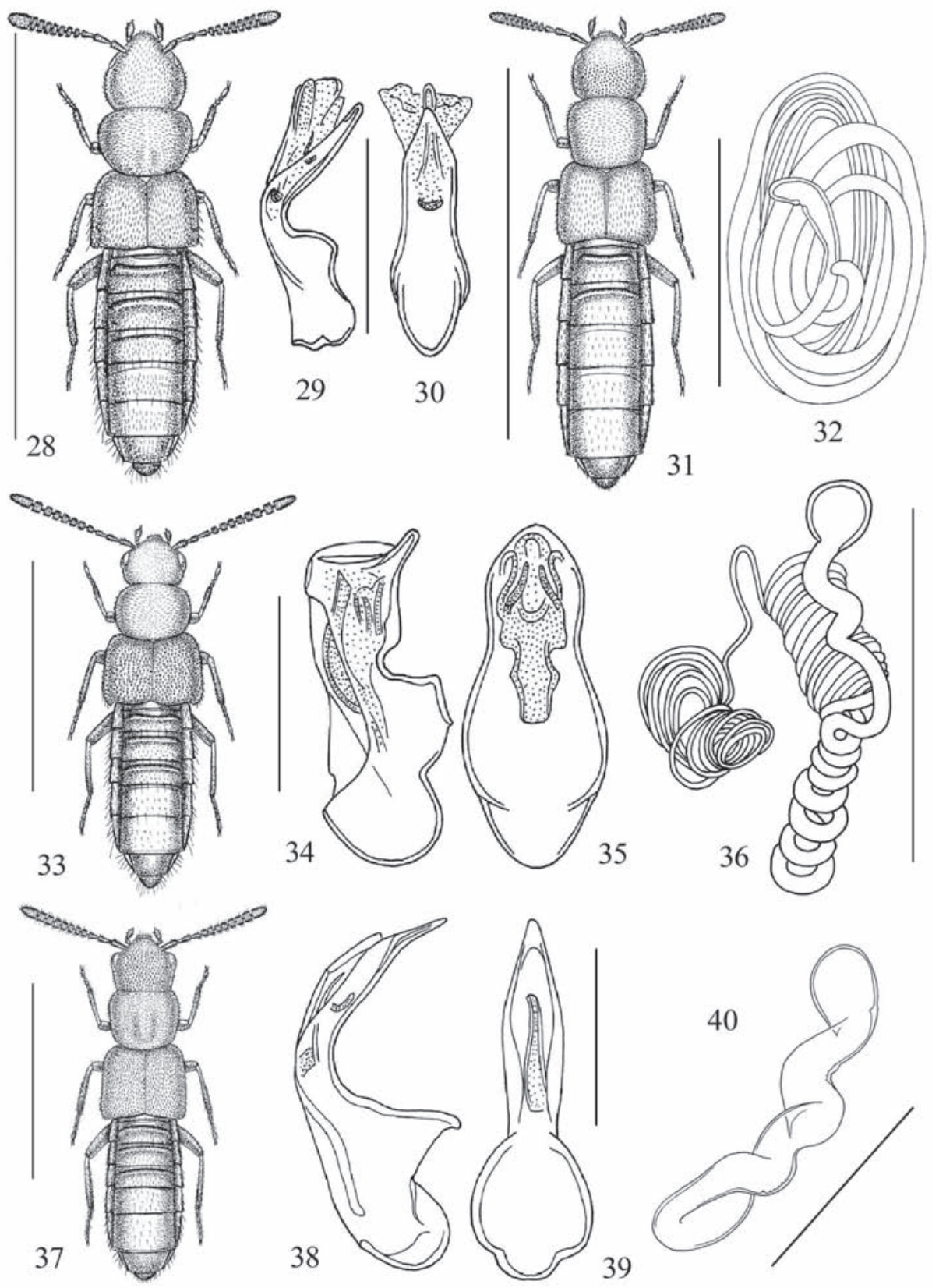

39

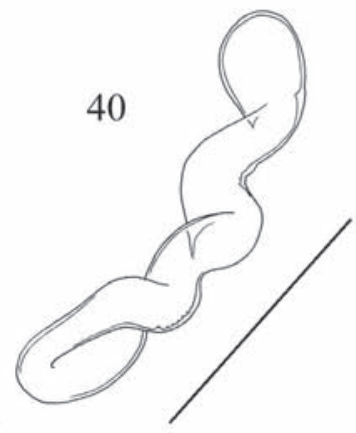

Figg. 28-40: Habitus, edeago in visione laterale e ventrale e spermateca. 28-30. Plesiomalota (Microusa) belemensis n. sp.; 31-32. Plesiomalota (Microusa) minima n. sp.; 33-36. Plesiomalota (Plesiomalota) tarapotensis n. sp.; 37-40. Plesiomalota (Plesiomalota) bradyporum n. sp. Habitus scala $1 \mathrm{~mm}$, altre scale 0,1 mm. 


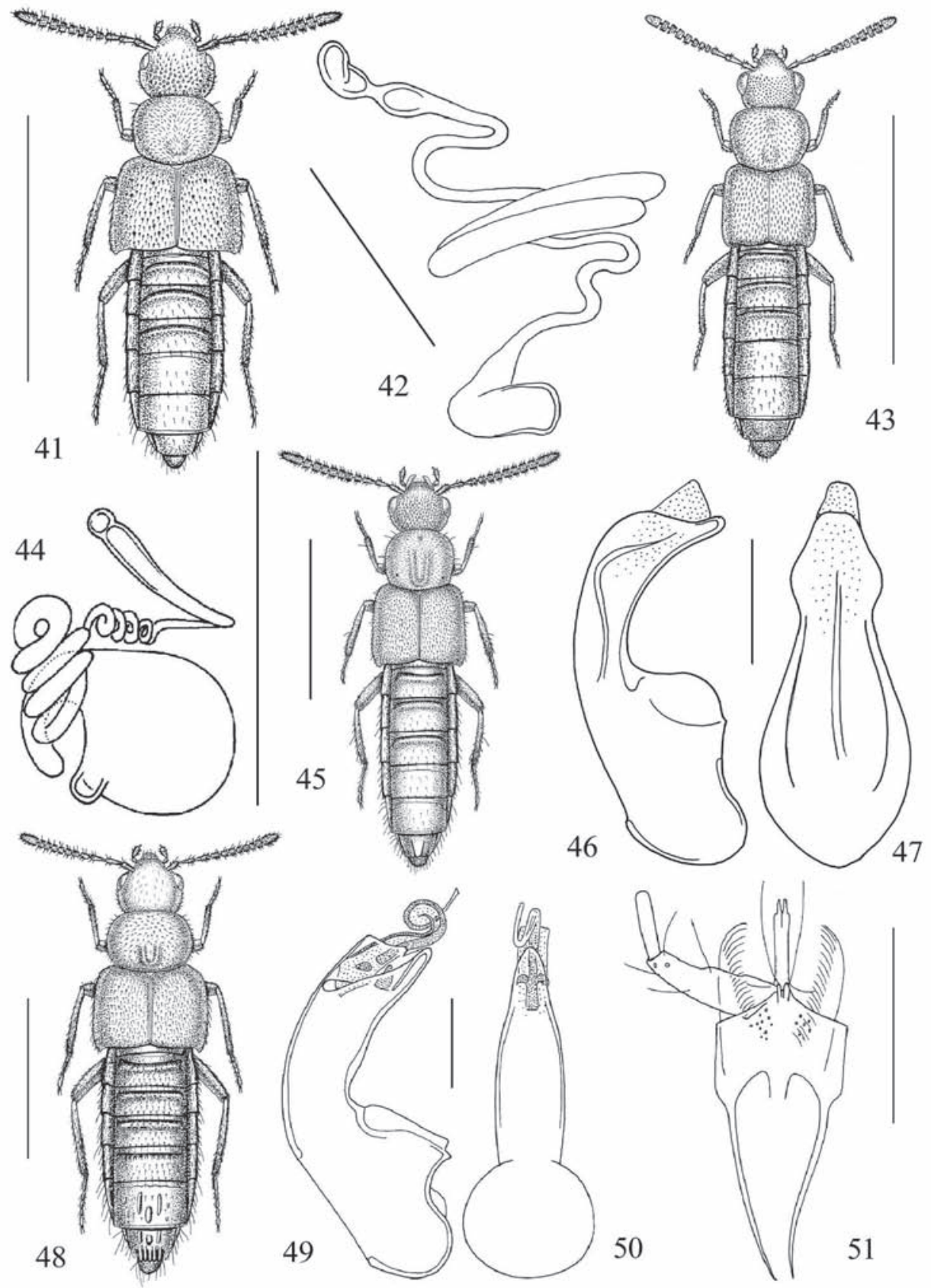

Figg. 41-51: Habitus, spermateca, edeago in visione laterale e ventrale e labio con palpo labiale. 41-42. Plesiomalota (Microchara) boliviana n. sp.; 43-44. Plesiomalota (Microusa) surinamensis n. sp.; 45-47. Plesiomalota (Plesiomalota) iguazuensis n. sp.; 48-51. Tropocoenonica catharinensis $n$. $s p$. Habitus scala $1 \mathrm{~mm}$, altre scale $0,1 \mathrm{~mm}$. 


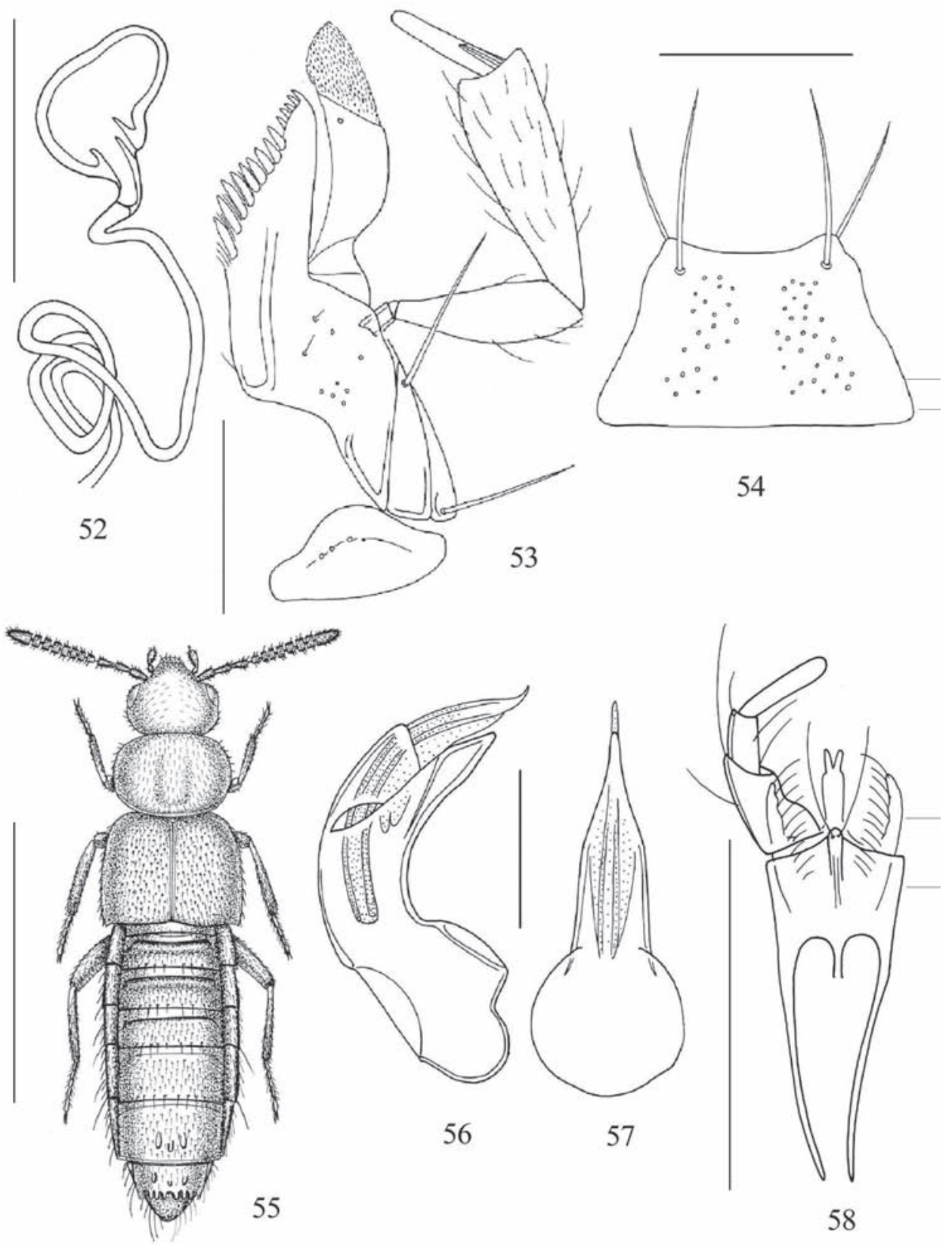

Figg. 52-58: Spermateca, maxilla con palpo mascellare, mento, habitus, edeago in visione laterale e ventrale e labio con palpo labiale. 52-54. Tropocoenonica catharinensis n. sp.; 55-58. Prosoponotha brasiliensis n. sp. Habitus scala $1 \mathrm{~mm}$, altre scale $0,1 \mathrm{~mm}$. 


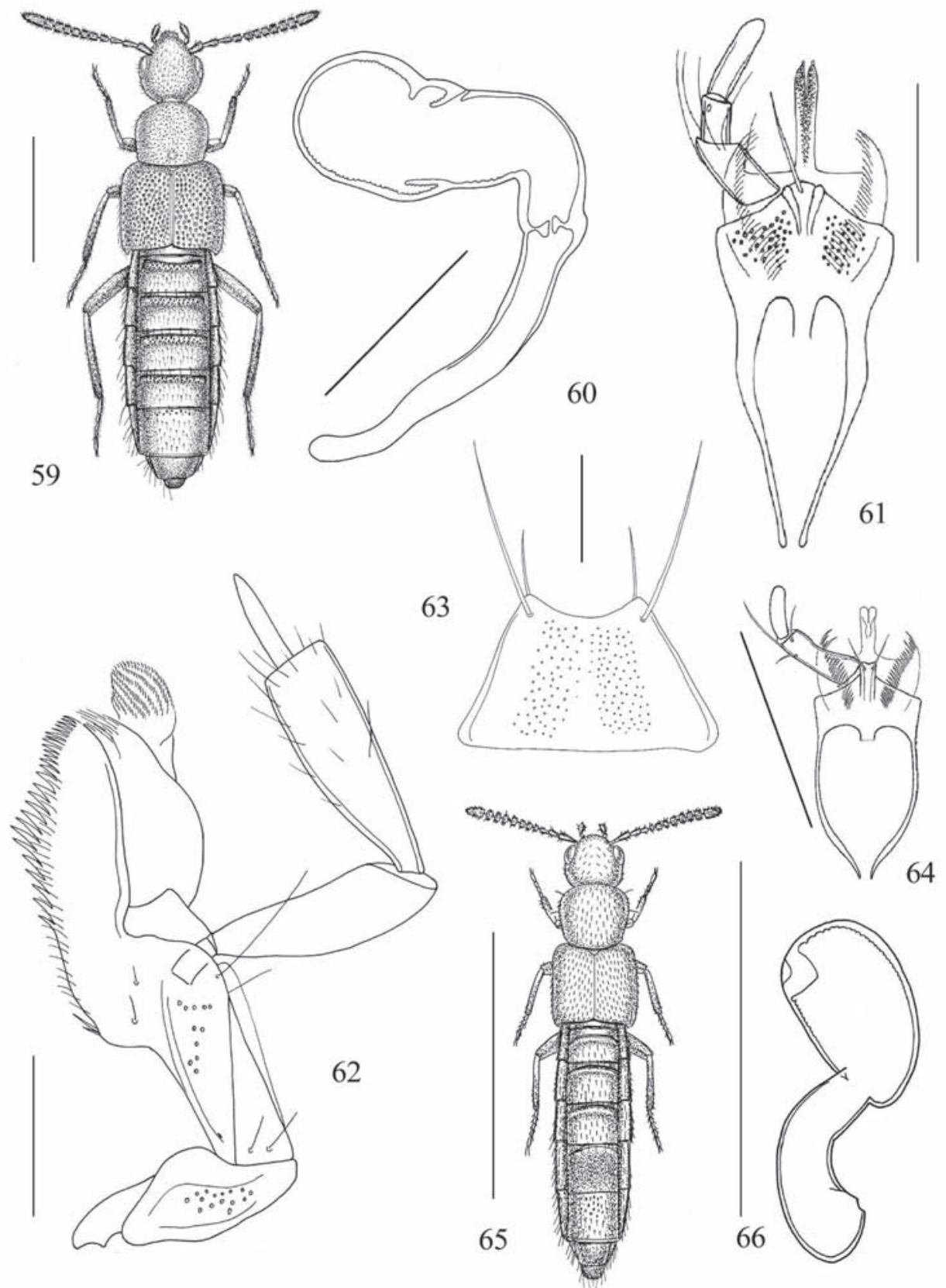

Figg. 59-66: Habitus, spermateca, labio con palpo labiale, mento e maxilla con palpo mascellare. 59-63. Dispachyglossa elegans n. sp.; 64. Coenonica puncticollis KraATZ; 65-66. Thecturota maculata n. sp. Habitus scala $1 \mathrm{~mm}$, altre scale $0,1 \mathrm{~mm}$. 


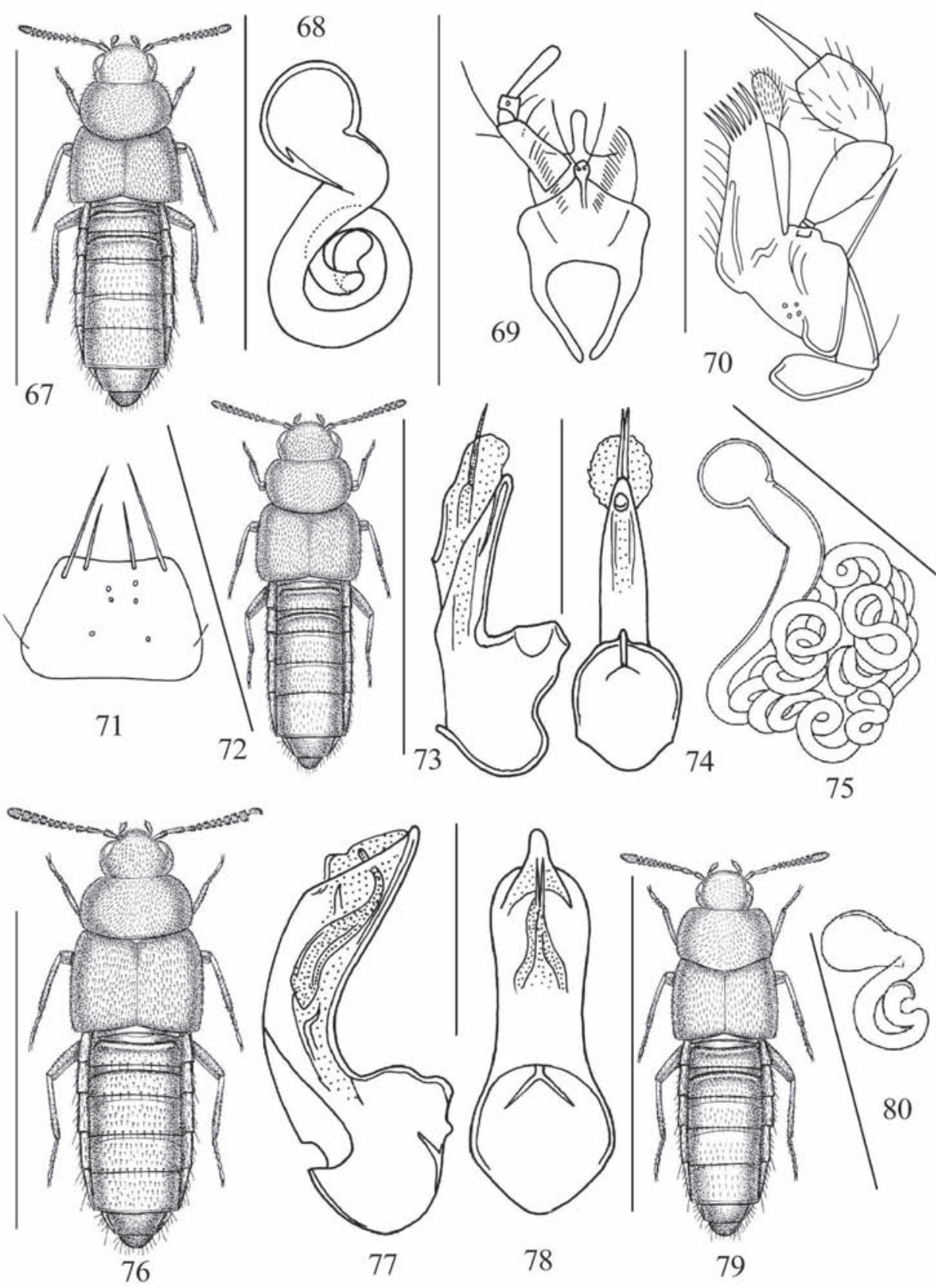

Figg. 67-80: Habitus, spermateca, labio con palpo labiale, maxilla con palpo mascellare, mento e edeago in visione laterale e ventrale. 67-71. Habramicrada boliviensis n. sp.; 72-75. Habramicrada anularis n. sp.; 76-78. Habramicrada peruviana n. sp.; 79-80. Habramicrada incaica n. sp. Habitus scala $1 \mathrm{~mm}$, altre scale $0,1 \mathrm{~mm}$. 


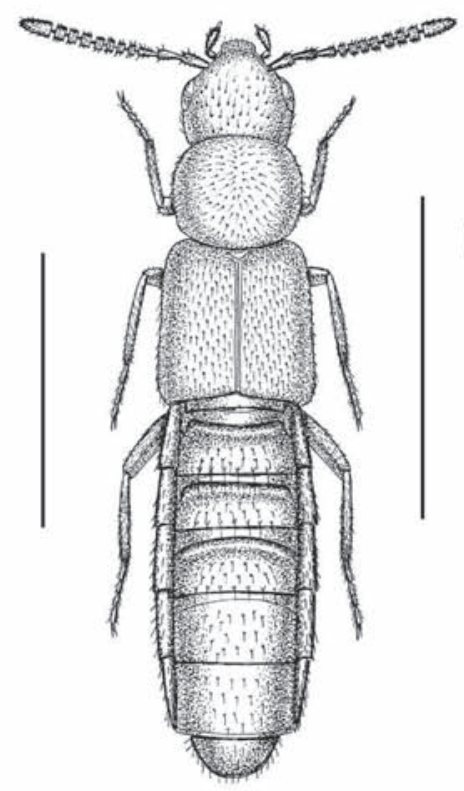

81

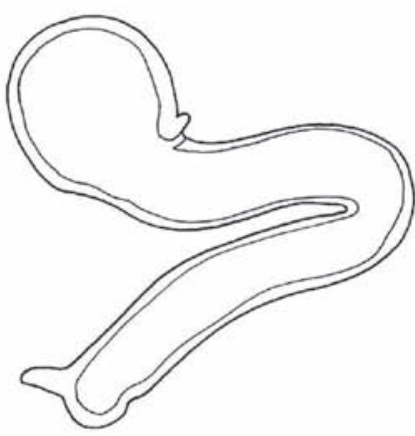

82
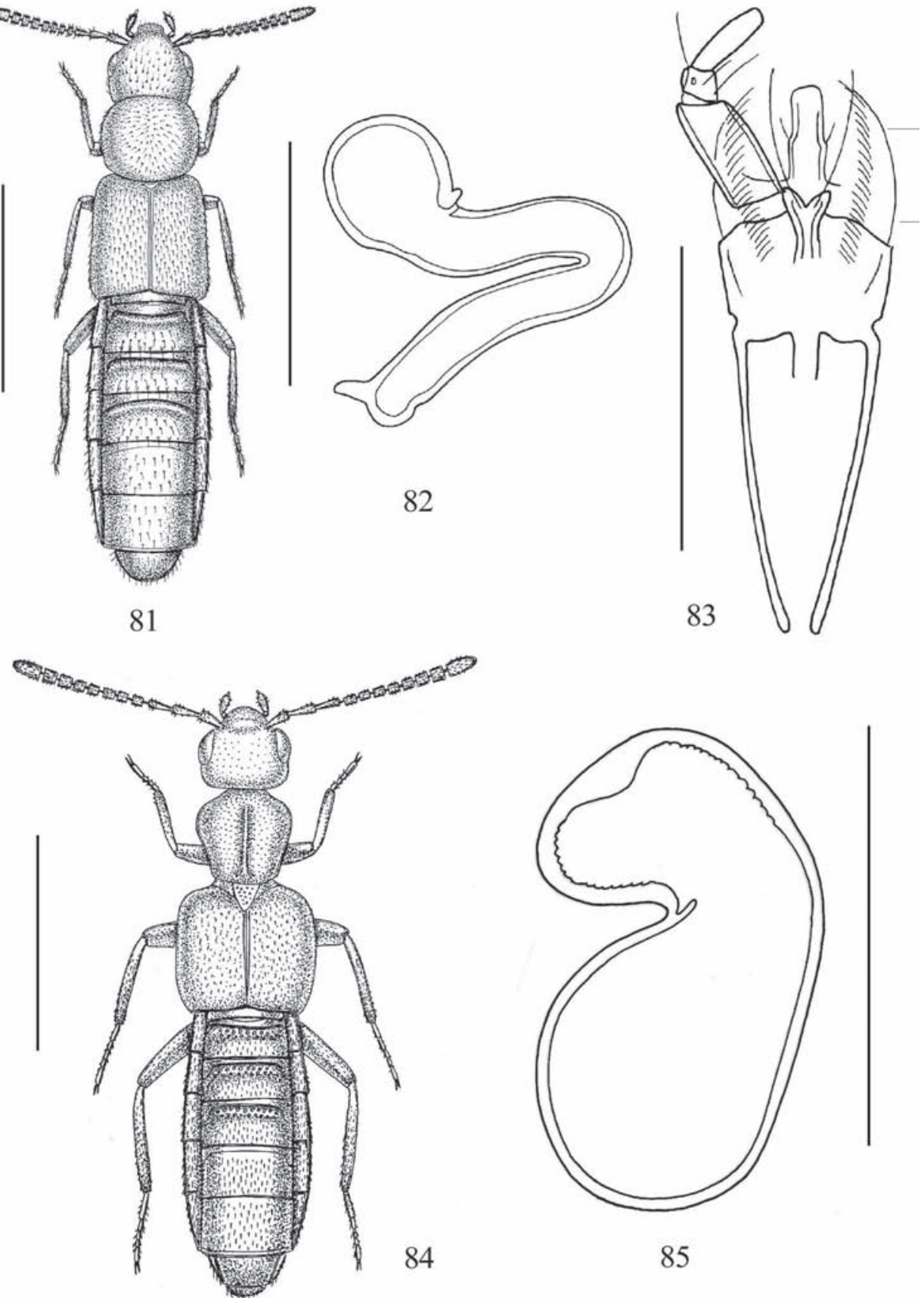

Figg. 81-85: Habitus, spermateca e labio con palpo labiale. 81-83. Pagla (Parallelopagla) levis n. sp.; 84-85. Falagria (Leptagria) boliviana n. sp. Habitus scala $1 \mathrm{~mm}$, altre scale $0,1 \mathrm{~mm}$. 

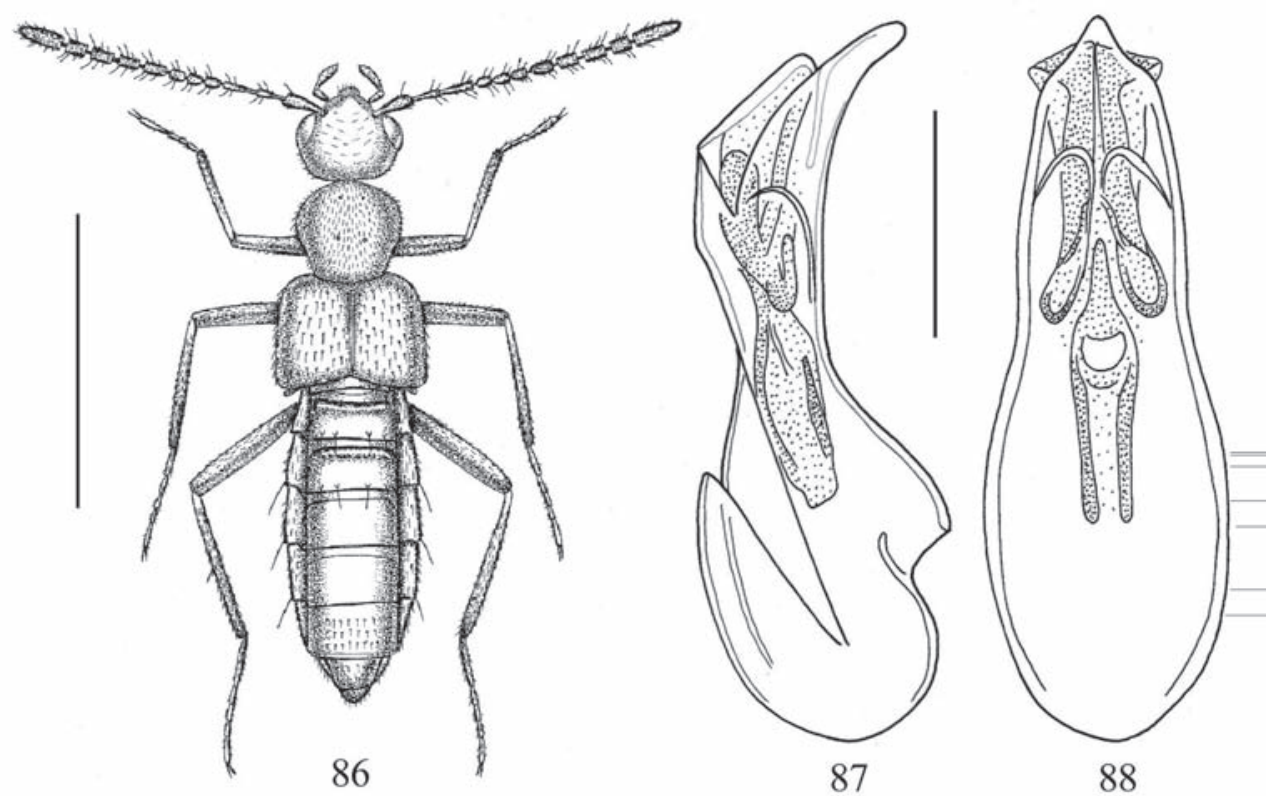

Figg. 86-88: Habitus e edeago in visione laterale e ventrale. 86-88. Meronera tersa $\mathrm{n}$. sp. Habitus scala $1 \mathrm{~mm}$, altra scala $0,1 \mathrm{~mm}$.

\section{Indirizzo dell'autore:}

Roberto Pace

Via Vittorio Veneto, 13

37032 Monteforte d'Alpone (Verona)

Italia

e-mail: pace.ent@tiscali.it

\section{Subject editor:}

Prof. Dr. B. Klausnitzer 